\title{
Neural Networks for Iris Recognition: Comparisons between LVQ and Cascade Forward Back Propagation Neural network Models, Architectures and Algorithm
}

\author{
Shivani Godara ${ }^{1}$, Dr. Rajeev Gupta ${ }^{2}$ \\ UCE, Rajasthan Technical University, Kota 324010, India
}

\begin{abstract}
In this research, Iris recognition is a proven, accurate means to identify people. In this paper, it includes the preprocessing system, segmentation, feature extraction and recognition. an iris recognition system was suggested based on two Artificial Neural Network (ANN) models separately: cascade forward (CFBPNN) and linear vector quantization (LVQ). Comparison results showed that linear vector quantization (LVQ) was the best training algorithm for the iris recognition system. Linear vector quantization (LVQ) is faster than cascade forward (CFBPNN).
\end{abstract}

Keywords: Biometrics, Cascade Forward back propagation neural network, Pattern Recognition, Canny edge detection, learning vector quantization neural network.

\section{INTRODUCTION}

There are variable ways of human verification throughout the world, as it is of great importance for all organizations, and different centers. Nowadays, the most important ways of human verification are recognition via DNA, face, fingerprint, signature, speech, and iris. Among all, one of the recent, reliable, and technological methods is iris recognition which is practiced by some organizations today, and its wide usage in the future is of no doubt.Iris is a non identical organism made of colorful muscles including robots with shaped lines. These lines are the main causes of making every one's iris nonidentical. Even the irises of a pair of eyes of one person are completely different from one another. Even in the case of identical twins irises are completely different. Each iris is specialized by very narrow lines, rakes, and vessels in different people. The precision of identification via iris is increased by using more and more details. It has been proven that iris patterns are never changed nearly from the time the child is one year old throughout all his life. Over the past few years there has been considerable interest in the development of neural network based pattern recognition systems, because of their ability to classify data. The iris images prepared as the database is in the form of PNG (portable network graphics) pattern, meanwhile they must be preprocessed through which the boundary of the iris is recognized and their features are extracted. For doing so, edge detection is done by the usage of canny approach.

\section{NEURAL NETWORK}

In this work two Neural Network structure is used, which is cascade forward (CFBPNN) and Learning Vector Quantization Neural Network. A brief overview of this network is given below.

\subsection{LEARNING VECTOR QUANTIZATION}

Learning Vector Quantization (LVQ) is a supervised version of vector quantization, similar to Self organizing Maps (SOM) based on work of Linde et al, Gray and Kohonen. It can be applied to pattern recognition, multi-class classification and data compression tasks, e.g. speech recognition, image processing or customer classification. As supervised method, LVQ uses known target output classifications for each input pattern of the form. LVQ algorithms do not approximate density functions of class samples like Vector Quantization or Probabilistic Neural Networks do, but directly define class boundaries based on prototypes, a nearest-neighbor rule and a winner-takes-it-all paradigm. The main idea is to cover the input space of samples with 'codebook vectors' (CVs), each representing a region labeled with a class. A CV can be seen as a prototype of a class member, localized in the centre of a class or decision region in the input space. A class can be represented by an arbitrarily number of CVs, but one $\mathrm{CV}$ represents one class only. In terms of neural networks a LVQ is a feed forward net with one hidden layer of neurons, fully connected with the input layer. 


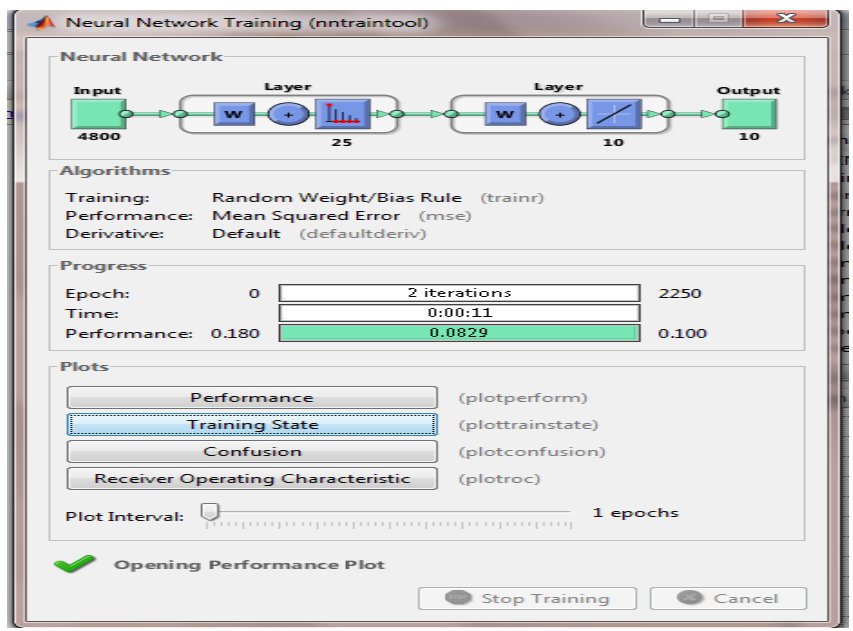

Fig: 1 Iris matching using LVQ neural network

A CV can be seen as a hidden neuron ('Kohonen neuron') or a weight vector of the weights between all input neurons and the regarded Kohonen neuron respectively. Learning means modifying the weights in accordance with adapting rules and, therefore, changing the position of a CV in the input space. Since class boundaries are built piecewise-linearly as segments of the mid-planes between CVs of neighboring classes, the class boundaries are adjusted during the learning process. The tessellation induced by the set of CVs is optimal if all data within one cell indeed belong to the same class. Classification after learning is based on a presented sample's vicinity to the CVs: the classifier assigns the same class label to all samples that fall into the same tessellation - the label of the cell's prototype (the CV nearest to the sample). The core of the heuristics is based on a distance function - usually the Euclidean distance is used - for comparison between an input vector and the class representatives. The distance expresses the degree of similarity between presented input vector and CVs. Small distance corresponds with a high degree of similarity and a higher probability for the presented vector to be a member of the class represented by the nearest CV. Therefore, the definition of class boundaries by LVQ is strongly dependent on the distance function, the start positions of CVs, their adjustment rules and the preselection of distinctive input features. Briefly explaining, this network has two layers: a layer of input neurons, and a layer of output neurons. The network is given by prototypes $\mathrm{W}=(\mathrm{w}(\mathrm{i}), \ldots, \mathrm{w}(\mathrm{n}))$. It changes the weights of the network in order to classify the data correctly. For each data point, the prototype (neuron) that is closest to it is determined (called the winner neuron). The weights of the connections to this neuron are then adapted, i.e. made closer if it correctly classifies the data point or made less similar if it incorrectly classifies it.

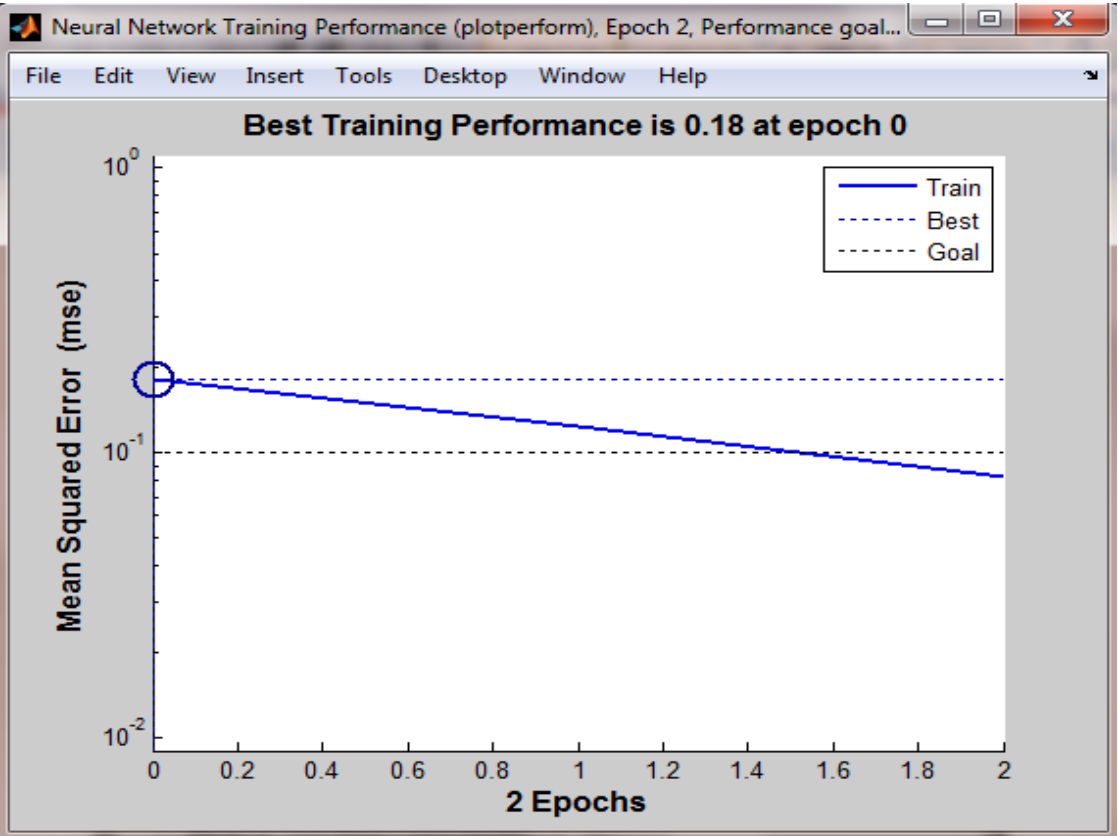

Fig 2: LVQ neural network training Performance 


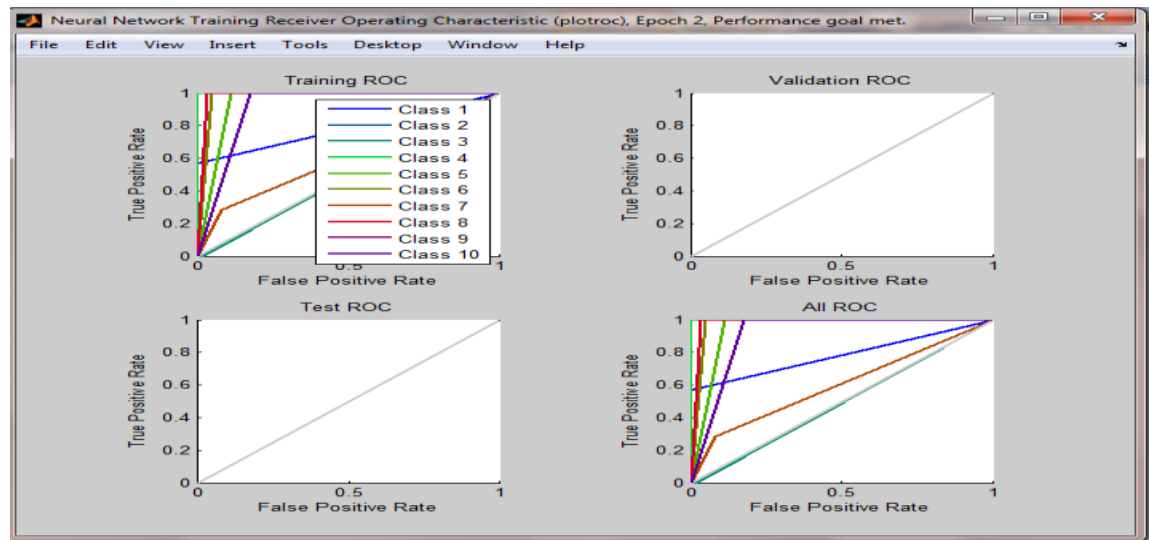

Fig 3: LVQ NN training Receiver Operating Characteristics

\subsection{FEED \& CASCADE - FORWARD BACK PROPAGATION}

Feed - Forward Back propagation neural network (FFBPNN) and Cascade Forward Back propagation neural network (CFBPNN) shown in Fig. [2]. A FFBPNN and CFBPNN have three layers: an input layer, hidden layer and an output layer. The neurons in the input layer only act as buffer for distributing the input signals to neuron in hidden layer. Each neuron in hidden layer sums up its input signal after weighting them and computes it outputs. Training a network consists of adjusting its weights using learning algorithms.

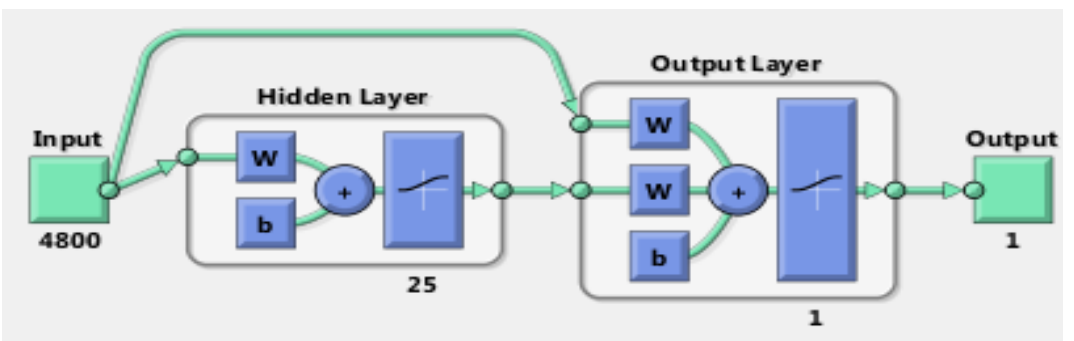

Fig 4: cascade-forward back propagation network.

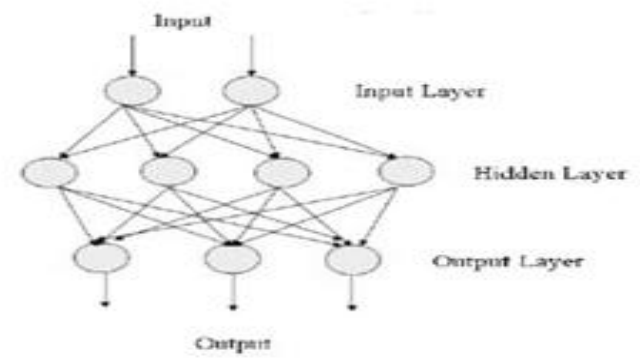

Fig 5: Architecture of cascade-forward back propagation network.

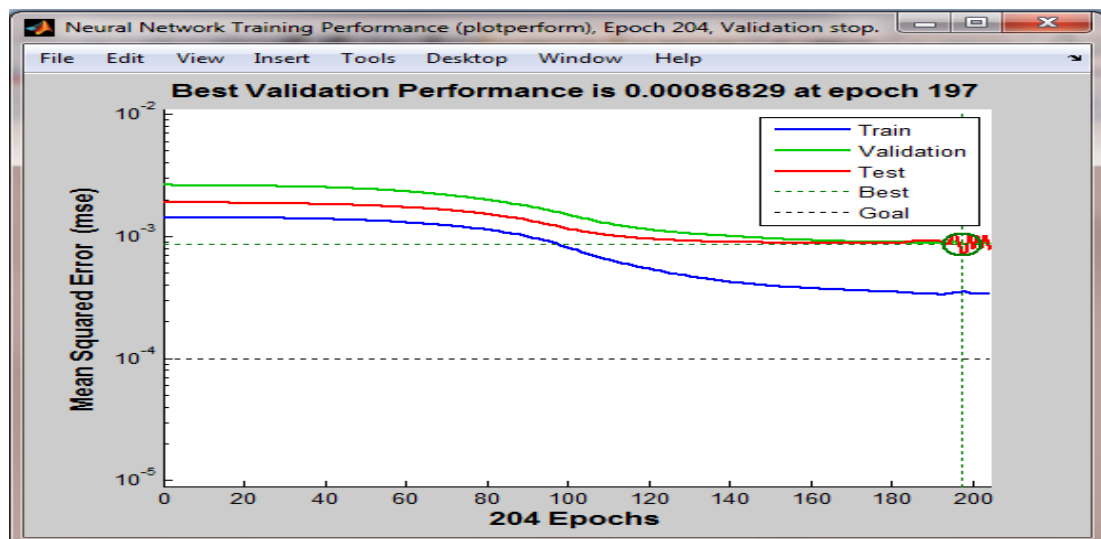

Fig 6: neural network training Performance 


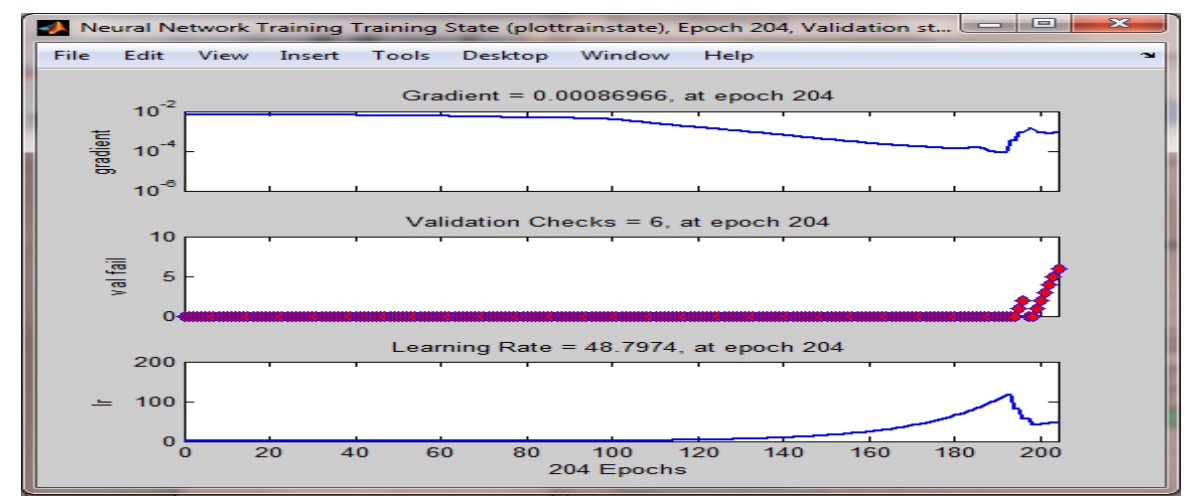

Fig 7: Neural network training state

III.

CONCLUSION

Main goal of this research paper is achieved by designing of efficient high-speed iris recognition system. In this paper, a two technique is proposed for iris verification. The classification is performed using LVQ Neural Network and cascade-forward back propagation network. Both neural network based approach is found to be a promising for iris recognition but LVQ neural network approach is less time consuming.

\section{REFERENCES}

[1]. M. Gopikrishnan, T.Santhanam, "Effect of different neural network on the accuracy in iris patterns recognition", International Journal of Reviews in Computing, Vol. 7 September 2011.

[2]. Bo Lu1, Jing-jing Wu, Yu Wang, "An iris recognition algorithm based on ICA and SOM neural network", CISP2010, pp 2246- 2448.

[3]. Victor-emil Neagoe, "New self-organizing maps with non-conventional metrics and their applications for iris recognition and automatic translation", proce 11th WSEAS International Conference on computers, Greece, July, 2007, pp 145-151.

[4]. Huiru Zheng, Haiying Wang," Improving pattern discovery and visualization with self-adaptive neural networks through data transformations", Springer, September 2011.

[5]. Venkata Rama Prasad Vaddella, Kurupati Rama," artificial neural networks for compression of digital images: a review ", 2009-2010 IJRIC\& LLS, pp 75-82.

[6]. Shylaja S.S., K.N.Balasubramanya, Murthy, S. Nataranjan, Nisecheth, Muthuraj R.,Ajay S," Feed forward neural network based eye localization and recognition using hough transform", IJACSA Vol. 2,No. 3 march 2011.

[7]. Mr.Amir M.Usman Wagdarikar, Mr.Patil B.G,Mrs.Dr. Shaila subbaraman ," Performance Evalution of IRIS Recognition Algorithms using Neural Network Classifier", 2010 IEEE, pp 146-149.

[8]. Thomas Heseltine, Nick Pears, Jim Austin, Zezhi Chen," Face Recognition: A Comparison of Appearance-Based Approaches " , Proc. VIIth Digital Image Computing: Techniques and Applications, Sun C., Talbot H., Ourselin S. and Adriaansen T. (Eds.), 10-12 Dec. 2003, Sydney ,pp 50-69.

[9]. M. Gopikrishnan, T.Santhanam, "Improved biometric recognition and indetification of human iris patterns using neural networks", 2005 - 2011 JATIT \& LLS, September 2011. Vol 31.

[10]. Shivani Godara, Dr. Rajeev Gupta, "Comparison of Different Neural Networks for Iris Recognition: A Review", IISTE, Vol 2, No.4, 2012, PP 8-15.

[11]. Shivani Godara, Ravindra Panchariya, "COMPARISON OF DIFFERENT NEURAL NETWORKS FOR IRIS RECOGNITION", [IJESAT] INTERNATIONAL JOURNAL OF ENGINEERING SCIENCE \& ADVANCED TECHNOLOGY Volume-2, Issue-4, pp 1168 - 1172. 